\title{
Numerical Study on Natural Convection of Alumina-water Nanofluid in a Square Cavity with Two Localized Heat Sources on Adjacent Surfaces
}

\author{
Farooq Shaik $^{1 *}$, Vinay Kumar Domakonda ${ }^{1}$ \\ ' Department of Mechanical Engineering, Vignan's Foundation for Science Technology and Research, Vadlamudi, Guntur 522213, \\ Andhra Pradesh, India \\ *Corresponding author, e-mail: skf_mech@vignan.ac.in
}

Received: 27 June 2020, Accepted: 05 March 2021, Published online: 28 June 2021

\begin{abstract}
In the present work, results of a numerical study carried out using finite volume method, to investigate the fluid flow and heat transfer characteristics of Alumina $\left(\mathrm{Al}_{2} \mathrm{O}_{3}\right)$ nanoparticles in the base fluid (water) in a square cavity under natural convection mode are presented. The Semi Implicit Method for Pressure Linked Equations (SIMPLE) algorithm was used to solve the discretized momentum and energy equations. Constant temperature heat sources of same strength are placed on bottom and left vertical surfaces whereas the right surface was kept cold, while the top surface was maintained as adiabatic. The impact of Rayleigh number (RaN) (1000 to $10^{6}$ ) and nanoparticles volume fraction ( $\Phi=0 \%, 5 \%, 10 \%, 15 \%$ and $20 \%$ ) on fluid and heat flow characteristics were numerically investigated and presented in the form of streamlines, isothermal lines, mid line horizontal and vertical velocity components, local Nusselt number $\left(\mathrm{Nu}_{\text {loc }}\right)$ and average Nusselt number $\left(\mathrm{Nu}_{\text {avg }}\right)$. The obtained results indicate, for lower RaN (i.e; 10³), conduction dominates over convection near heated surfaces and results in lower fluid velocities and poor heat transfer. For higher values of $\operatorname{RaN}\left(\operatorname{RaN}=10^{5}\right.$ and $\left.10^{6}\right)$ and volume fraction of nanoparticles, there was a significant increase in mid horizontal and vertical velocity components, $\mathrm{Nu}_{\text {loc }}$ and $\mathrm{Nu}_{\text {avg }}$ due to increase in convective heat transfer and thermal conductivity of nanofluid.
\end{abstract}

Keywords

Nusselt number, nanofluid, alumina nanoparticles, Rayleigh number, nanoparticles volume fraction

\section{Introduction}

Due to wide variety applications of natural convective heat transfer such as electronics cooling, insulation of nuclear reactors, heat exchangers, micro channel heat sinks, solar collectors, crystal growth in liquids and many other industrial and engineering applications, it has been a major area of research for scientists and engineers for many years. Cooling fluids used in heat transfer applications such as water, ethylene glycol, toluene, mineral oils, etc. possess poor thermal conductivity which impose limitations on their wider range of applications. There have been numerous strategies being tested to find out alternatives for these conventional cooling mediums and one among them is nanofluids. Available literature in the area concerned indicates, with theaddition of nanosize particles of metals, metal oxides, nitrides to the base fluids, heat carrying capacity of the base fluid increasesdue to increase in the thermal conductivity. Nanofluids are dilute liquid suspensions of nanoparticles with diameter lesser than
$100 \mathrm{~nm}$ suspending stable and uniform in a base fluid [1]. Keblinski et al. [2] have reported that thermal conductivity increases with decrease in grain size of nanoparticles and explained the influential mechanisms responsible for enhanced heat conduction in nanofluids. Xuan and Li [3] developed methods for preparation of nanofluids and discussed that the shape, dimensions, volume fraction and properties of nanoparticles affects the thermal conductivity of nanofluids. Jang and Choi [4] theoretically investigated a model of thermal conductivity and reported that as the volume fraction of nanoparticles in the base fluid increases, the ratio of thermal conductivity of nanofluid to its base fluid and the ratio of viscosity of nanofluid to the base fluid increases. Jang and Choi [5] developed a theoretical model to understand the importance of Brownian motion of nanoparticles at the micro and nanoscale level and also predicts the particle-size and temperature-dependent conductivity of nanofluids. Xie et al. [6] prepared 
different suspensions of $\mathrm{Al}_{2} \mathrm{O}_{3}$ nanoparticles for their experimental study and reported that increase in thermal conductivity of nanofluid is highly dependent on specific surface area of nanoparticles. And also discussed that thermal conductivity ratio decrease with increase in thermal conductivity of base fluid with the same nanoparticles. Wang et al. [7] numerically investigated the effect of rod and sphere shaped $\mathrm{Al}_{2} \mathrm{O}_{3}$ nanoparticles on convective heat transfer inside a cubical enclosure and reported, for Rayleigh number more than 10000, average Nusselt number decreases with increase in volume fraction of nanoparticles for rod shaped particles where as it increases for spherical shaped particles. Khalili et al. [8] performed computational investigation of alumina nanoparticles $(1 \%$, $2 \%, 5 \%$ ) at Rayleigh numbers $10^{3}$ and $10^{5}$ through hot and cold tubes of different diameters and reported that average Nusselt number increases with increase in volume fraction of nanoparticles but reported decrease in nanofluid heat transfer. Bondarenko et al. [9] performed numerical simulation of natural convective heat transfer consisting of heat conducting and heat generating source placed in alumina-water nanofluid inside the cavity. Their results showed that heat conducting and heat generating element of poor thermal conductivity can be cooled more effectively by nanofluids. Rashidi et al. [10] have investigated the flow and heat transfer characteristics of $\mathrm{Al}_{2} \mathrm{O}_{3}$ nanofluid filled in a square cavity for different aspect ratios and volume fraction of nanoparticles. It has been reported that vertical velocity component of nanaofluid at mid horizontal line of the cavity decreases with increase in volume fraction of nanoparticles from $3 \%$ to $9 \%$ at $\mathrm{RaN}=10^{4}$. Though the applications of heat sources on adjacent sides of the wall are wide, as in case of natural convective cooling of electronic devices, the literature available in this regard using nanoparticles in base fluid is very limited. In the present work, the impact of RaN and volume fraction of nanofluids on fluid flow and heat transfer inside the square cavity filled with $\mathrm{Al}_{2} \mathrm{O}_{3}$-Water nanofluid with adjacent localized heat sources is numerically investigated using FVM for steady flow laminar natural convection.

\subsection{Description of the problem}

The schematic representation of square cavity filled with $\mathrm{Al}_{2} \mathrm{O}_{3}$-Water nanofluid is presented Fig. 1. Two constant temperature hot sources, $T_{h}$ are located on two adjacent surfaces (Bottom and Left vertical wall), while vertical right surface was maintained at low temperature $T_{c}$, and the top surface is insulated. The assumptions made

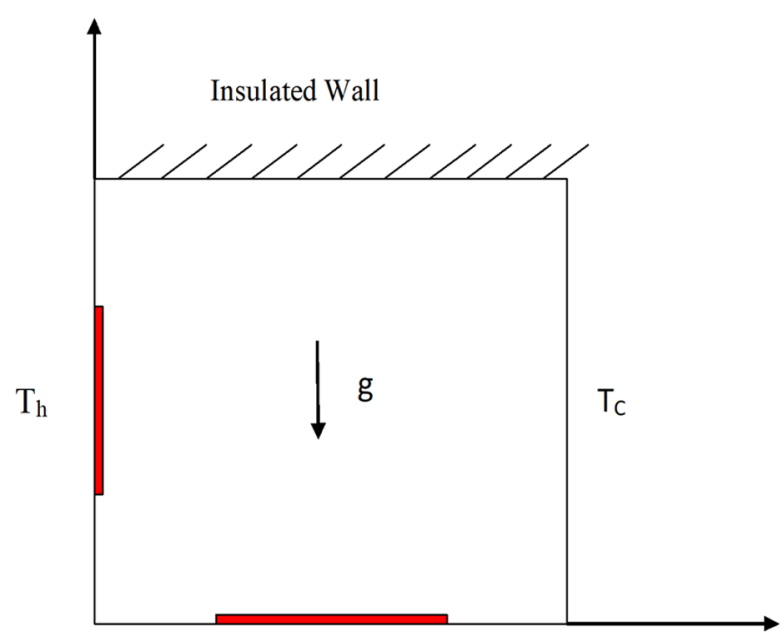

$T_{h}$

Fig. 1 Schematic view of the problem with heat sources on bottom and left surfaces

for the nanofluid flow and heat transfer inside the cavity are steady, two dimensional, laminar, incompressible and Newtonian fluid. Boussinesq approximation is considered to account for density variation of nanofluids and the other properties are assumed to be constant.

\section{Mathematical formulation of the problem}

The following are the dimensionless terms are used to obtain dimensionless governing equations are

$$
\begin{aligned}
& X=x / L, Y=y / L, U=\frac{u L}{\alpha_{f}}, V=\frac{v L}{\alpha_{f}}, P=\frac{p L^{2}}{\rho_{n f \alpha_{f}^{2}}}, \\
& \theta=\frac{\left(T-T_{c}\right)}{\left(T_{h}-T_{c}\right)} .
\end{aligned}
$$

The dimensionless fluid flow and energy equations are expressed as follows:

$$
\frac{\partial U}{\partial X}+\frac{\partial V}{\partial Y}=0
$$

$U \frac{\partial U}{\partial X}+V \frac{\partial U}{\partial Y}=-\frac{\partial P}{\partial X}+\frac{\mu_{n f}}{\rho_{n f} \alpha_{f}}\left(\frac{\partial^{2} U}{\partial X^{2}}+\frac{\partial^{2} U}{\partial Y^{2}}\right)$

$U \frac{\partial V}{\partial X}+V \frac{\partial V}{\partial Y}=-\frac{\partial P}{\partial Y}+\frac{\mu_{n f}}{\rho_{n f} \alpha_{f}}\left(\frac{\partial^{2} V}{\partial X^{2}}+\frac{\partial^{2} V}{\partial Y^{2}}\right)$

$+\frac{(\rho \beta)_{n f}}{\rho_{n f} \beta_{n f}} \operatorname{RaN} \operatorname{Pr} \theta$

$$
U \frac{\partial \theta}{\partial X}+V \frac{\partial \theta}{\partial Y}=\frac{\alpha_{n f}}{\alpha_{f}}\left[\left(\frac{\partial^{2} \theta}{\partial X^{2}}+\frac{\partial^{2} \theta}{\partial Y^{2}}\right)\right]
$$


$\operatorname{RaN}=\frac{g \beta_{f} L^{3}\left(T_{h}-T_{c}\right)}{v_{f} \alpha_{f}}$

$\operatorname{Pr}=\frac{v_{f}}{\alpha_{f}}$

The density, diffusivity, heat capacitance, coefficient of thermal expansion, thermal conductivity of nanofliuds [11] and viscosity [12] (Table 1) are determined by

$\rho_{n f}=(1-\phi) \rho_{f}+\phi \rho_{p}$

$\alpha_{n f}=k_{n f} /(\rho C)_{n f}$

$(\rho C)_{n f}=(1-\phi)(\rho C)_{f}+\phi(\rho C)_{p}$

$(\rho \beta)_{n f}=(1-\phi)(\rho \beta)_{f}+\phi(\rho \beta)_{p}$

$\mu_{n f}=\frac{\mu_{f}}{(1-\phi)^{2.5}}$

$k_{n f}=k_{f}\left[\frac{\left(k_{p}+2 k_{f}\right)-2 \phi\left(k_{f}-k_{p}\right)}{\left(k_{p}+2 k_{f}\right)+\phi\left(k_{f}-k_{p}\right)}\right]$.

The local and $\mathrm{Nu}_{\text {avg }}$ on the bottom surface is calculated by using the following expressions:

$$
\begin{aligned}
& \mathrm{Nu}_{\mathrm{loc}}=-\left.\frac{k_{n f}}{k_{f}} \frac{\partial \theta}{\partial Y}\right|_{Y=0} \\
& \mathrm{Nu}_{\mathrm{avg}}=\int_{0}^{1} \mathrm{Nu}_{\mathrm{loc}} \mathrm{d} X .
\end{aligned}
$$

The local and $\mathrm{Nu}_{\mathrm{avg}}$ on the left surface is calculated by using the following expressions:

$$
\begin{aligned}
& \mathrm{Nu}_{\mathrm{loc}}=-\left.\frac{k_{n f}}{k_{f}} \frac{\partial \theta}{\partial X}\right|_{X=0} \\
& \mathrm{Nu}_{\mathrm{avg}}=\int_{0}^{1} \mathrm{Nu}_{\mathrm{loc}} \mathrm{d} Y .
\end{aligned}
$$

Table 1 Thermo physical properties of Water and $\mathrm{Al}_{2} \mathrm{O}_{3}$-nanoparticles [9]

\begin{tabular}{lcccc}
\hline & $\rho\left(\mathrm{kg} / \mathrm{m}^{3}\right)$ & $C(\mathrm{~J} / \mathrm{kg} \mathrm{K})$ & $k(\mathrm{~W} / \mathrm{m} \mathrm{K})$ & $\beta(1 / \mathrm{K})$ \\
\hline Water & 997.1 & 4179 & 0.613 & $2.1 \times 10^{-4}$ \\
$\mathrm{Al}_{2} \mathrm{O}_{3}$ & 3970 & 765 & 40 & $0.85 \times 10^{-5}$ \\
\hline
\end{tabular}

\subsection{Boundary conditions}

The non-dimensional boundary conditions along the walls of the square cavity are applied as:

- At left surface $(X=0$ and $0 \leq Y \leq 1) U=V=\theta=0$, whereas along the heat source, $\theta=1$

- At right surface $(X=1$ and $0 \leq Y \leq 1) U=V=\theta=0$.

- At top surface $(Y=1$ and $0 \leq X \leq 1) U=V=\frac{\partial \theta}{\partial Y}=0$.

- At bottom surface $(Y=0$ and $0 \leq X \leq 1) U=V=\theta=0$, whereas along the heat source, $\theta=1$.

\section{Computational technique and code validation}

A Finite Volume Method (FVM) was used to discretize non-dimensional fluid flow and energy. In order to link the continuity and momentum equations, the SIMPLE algorithm was used. The convective and diffusive terms in the momentum and energy equations were handled with Quadratic Upstream Interpolation for Convective Kinematics (QUICK) scheme. The discretized linear equations were solved by Tri-Diagonal Matrix Algorithm (TDMA) method. The converging criterion is taken less than $10^{-8}$ for all the residuals of governing equations. The present developed $\mathrm{C}++$ numerical code was validated (Table 2) in terms of $\mathrm{Nu}_{\text {avg }}, U_{\max }, V_{\max }, U$ and $V$ velocity contour maps by varying RaN for natural convection heat

\begin{tabular}{|c|c|c|c|}
\hline & Present code & Benchmark results [13] & $\begin{array}{l}\text { Markatos and } \\
\text { Pericleous [14] }\end{array}$ \\
\hline \multicolumn{4}{|c|}{$\mathrm{RaN}=10^{3}$} \\
\hline $\mathrm{Nu}_{\mathrm{avg}}$ & 1.134 & 1.11 & 1.108 \\
\hline$U_{\max }$ & 3.640 & 3.649 & 3.544 \\
\hline$V_{\max }$ & 3.60 & 3.697 & 3.593 \\
\hline \multicolumn{4}{|c|}{$\mathrm{RaN}=10^{4}$} \\
\hline $\mathrm{Nu}_{\text {avg }}$ & 2.102 & 2.243 & 2.201 \\
\hline$U_{\max }$ & 16.10 & 16.17 & 16.18 \\
\hline$V_{\max }$ & 19.41 & 19.61 & 19.44 \\
\hline \multicolumn{4}{|c|}{$\mathrm{RaN}=10^{5}$} \\
\hline $\mathrm{Nu}_{\text {avg }}$ & 4.53 & 4.519 & 4.430 \\
\hline$U_{\max }$ & 35.95 & 34.73 & 35.73 \\
\hline$V_{\max }$ & 68.99 & 68.59 & 69.08 \\
\hline \multicolumn{4}{|c|}{$\mathrm{RaN}=10^{6}$} \\
\hline $\mathrm{Nu}_{\text {avg }}$ & 8.99 & 8.799 & 8.754 \\
\hline$U_{\max }$ & 64.50 & 64.63 & 68.81 \\
\hline$V_{\max }$ & 215.35 & 217.36 & 221.8 \\
\hline
\end{tabular}
transfer. It was done considering air as a medium inside the square cavity in which the left wall was maintained hot

Table 2 Validation of present developed code with benchmark results. 
and right wall was maintained at low temperature while the top and bottom walls were adiabatic. The obtained results were in good agreement in terms of average Nusselt number, $U_{\max }, V_{\max }, U$ and $V$ velocity contour maps with the work reported by $[13,14]$ as shown in Figs. 2 and 3.

\subsection{Grid independence study}

In order to determine accurate grid size for the developed numerical code, grid independence test was performed for the case of $5 \%$ volume fraction of $\mathrm{Al}_{2} \mathrm{O}_{3}$-nanoparticles $(\Phi=5 \%)$ in the base fluid. Different uniform grids namely, $20 \times 20,40 \times 40,60 \times 60,80 \times 80,100 \times 100$ were tested for horizontal and vertical velocity components at mid horizontal and vertical lines at $\mathrm{RaN}=10^{4}$. It was observed from Fig. 4 (a) and (b) that both mid line $U$ and $V$ velocity components remained nearly same from grids $60 \times 60$ to $100 \times 100$. Hence $60 \times 60$ grid was chosen for rest of the study.
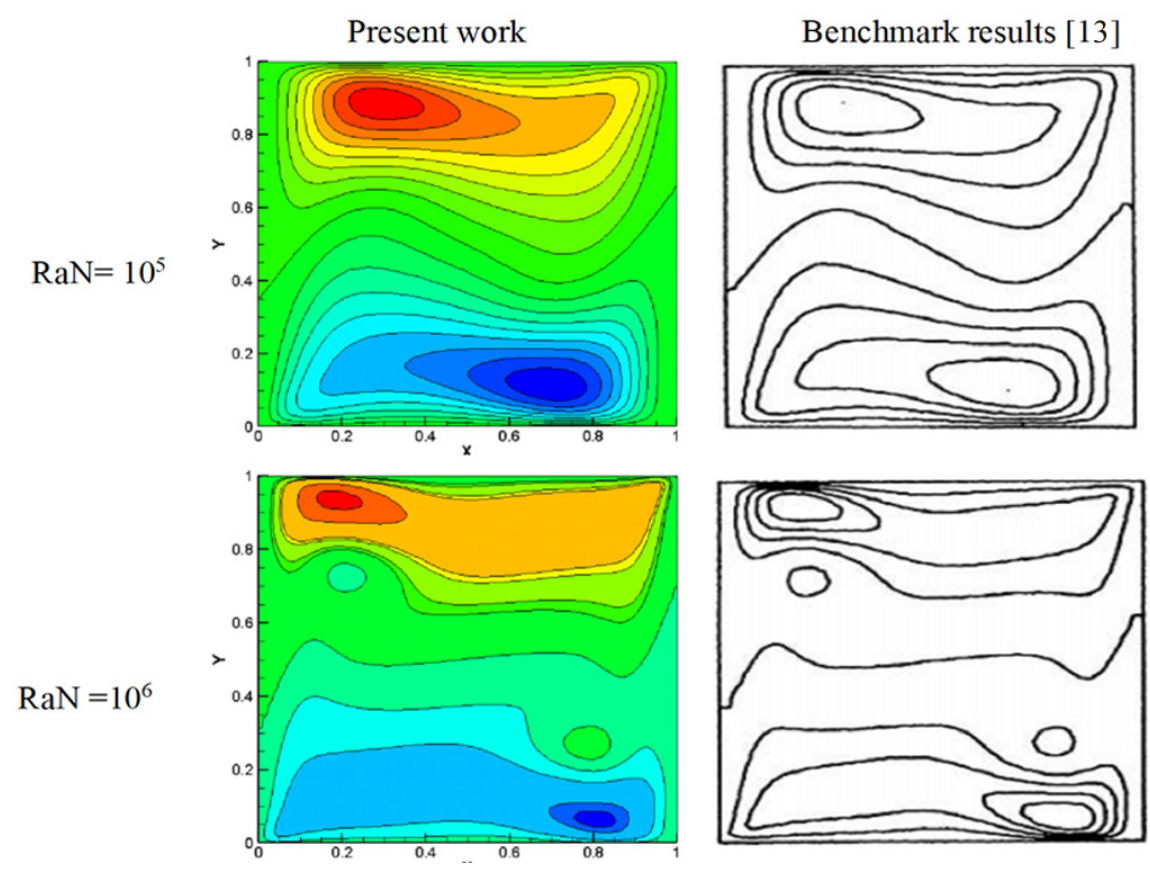

Fig. 2 Comparison of $U$-velocity contour maps with benchmark results [13] $\left(\mathrm{RaN}=10^{5}\right.$ and $\left.10^{6}, \operatorname{Pr}=0.71\right)$

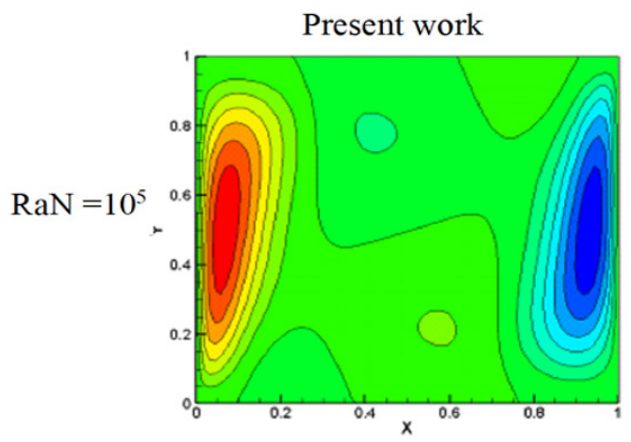

Benchmark results [13]
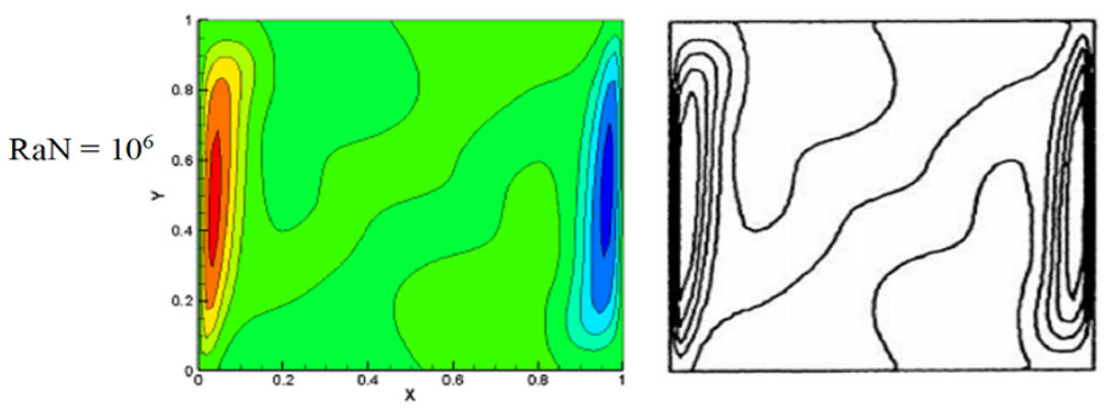

Fig. 3 Comparison of $V$-velocity contour maps with benchmark results [13] $\left(\mathrm{RaN}=10^{5}\right.$ and $\left.10^{6}, \operatorname{Pr}=0.71\right)$ 


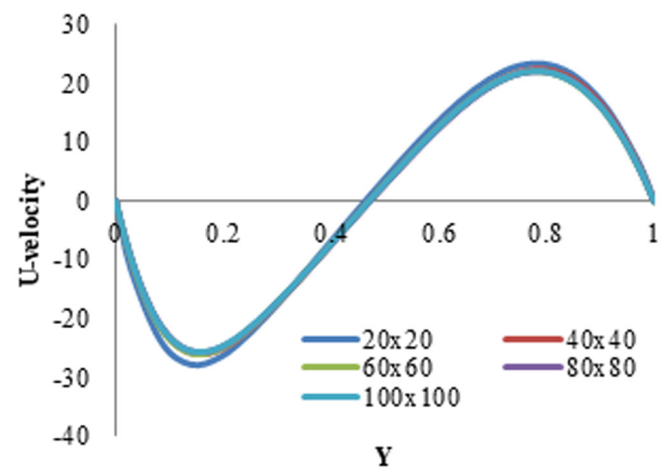

(a)

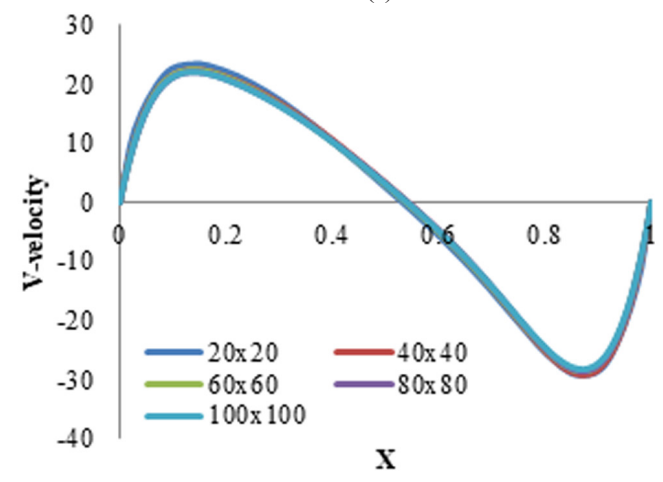

(b)

Fig. 4 Grid independence results at $\Phi=5$ percent, $\mathrm{RaN}=10^{4}$ (a) $U$-horizontal velocity along mid vertical line. (b) $V$-vertical velocity along mid horizontal line

\section{Results and discussion}

In this section, natural convection heat transfer and fluid flow of $\mathrm{Al}_{2} \mathrm{O}_{3}$-water nanofluid was numerically investigated for a steady, two-dimensional laminar flow. Two localized constant temperature heat sources of same magnitude were placed on the adjacent surfaces (bottom and left) of square cavity. The results were presented mainly in terms of impact of RaN and volume fraction of nanoparticles on streamlines, mid line horizontal and vertical fluid velocities, local and average Nusselt number over the heated surfaces inside the cavity. The value of RaN was varied from 1000 to $10^{6}$ and the volume fraction of $\mathrm{Al}_{2} \mathrm{O}_{3}$-nano sized particles was varied from 0 to $20 \%(\varphi=0 \%, 5 \%, 10 \%$, $15 \%$ and $20 \%$ ). For the entire study, Prandtl number of the fluid, Pr was taken as 6.2 and this number was chosen based on the literature available on similar work.

\subsection{Impact of RaN on streamlines}

Formations of streamlines in the cavity for different RaN are shown in Fig. 4. In this case, the cavity is filled with $\mathrm{Al}_{2} \mathrm{O}_{3}-\mathrm{NF}$ with $15 \%$ volume fraction of nanoparticles and the $\mathrm{RaN}$ varies from $10^{3}$ to $10^{6}$. Due to heat transfer through the heated surface, there was a change in density of the fluid inside the cavity, leading to buoyancy driven recirculation flows [14]. It can be observed from the Fig. 5 (a) to (d) that stream lines are developed over the heated surfaces with two contra-rotary vortices in the upper and lower left corners inside the cavity. The stream lines rise over the heated surfaces reaching towards top adiabatic surface and deflect downwards over the right cold surface and then forms a continuous circulating steady flow. At $\mathrm{RaN}=10^{3}$ streamlines of single vortex is formed in the center of the cavity. As the RaN increases from $10^{4}$ to $10^{5}$, the stream lines of central flow get distorted from circular vortices to elliptical shape at its center. At $\mathrm{RaN}=10^{6}$, streamlines in the center of the cavity gets further elongated and distorted resulting in the formation of multiple vortices inside it. Whereas the upper counter rotating vortices observed to be grows in size with increase in $\mathrm{RaN}$ from $10^{3}$ to $10^{6}$. This is due to higher fluid velocities that make large mass fluid to be driven towards right cold wall which creates empty space in the corners. And these counter rotating vortices rotate in direction opposite to that of main flow inside the cavity.

\subsection{Impact of RaN on isothermal lines}

Formation of isothermal lines due to two adjacent heated sources inside the cavity at $15 \%$ volume fraction of nanoprticles for different RaN can be seen in Fig. 6 (a) to (d). Due to the dominant effect of conduction heat transfer than the convection at the heated surfaces, especially at low $\mathrm{RaN}$ of $10^{3}$ and $10^{4}$, isothermal lines are formed nearly parallel to hot surfaces and to the walls resulting in poor heat transfer to the NF. However, it can be observed from Fig. 6 (c) and (d), for RaN values of $10^{5}$ to $10^{6}$, temperature gradient was high near the heated walls than at the center of the cavity and isothermal lines were distributed more randomly and also space between the isothermal lines and hot and cold walls decreased. This is due to the increased rate of heat transfer inside the cavity because of dominant role of convective heat transfer over the conduction. At $\mathrm{RaN}=10^{6}$, it can also be observed that the isothermal lines were formed nearly horizontal resulting in prevention of vertical motion of fluid in the central region, leading to thermal stratification for nearly $60 \%$ width of the cavity, whereas higher vertical velocities were recorded near vertical surfaces.

\subsection{Impact of volume fraction of nanoparticles on horizontal component of velocity, $\boldsymbol{U}$}

The impact of volumetric percentage of $\mathrm{Al}_{2} \mathrm{O}_{3}$ nanoparticles on horizontal velocity components, $U$, along the mid vertical line inside the square cavity at different $\mathrm{RaN}$ is shown in Fig. 7. It is illustrated from Fig. 7 (a) that at low 


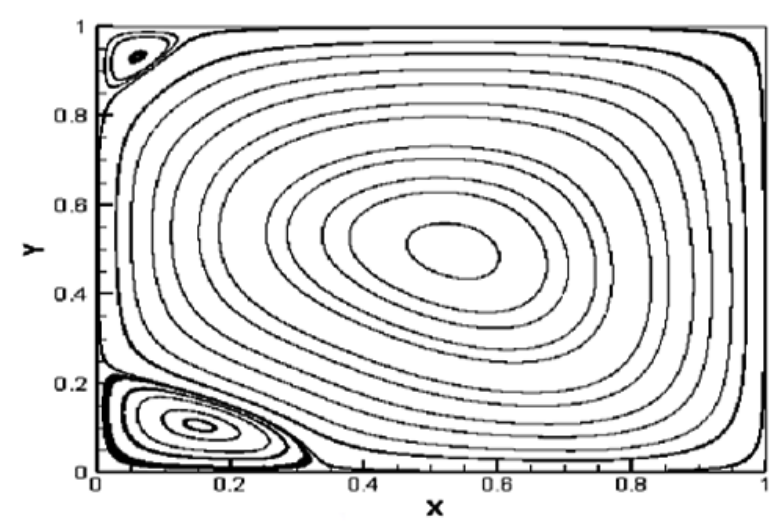

(a)

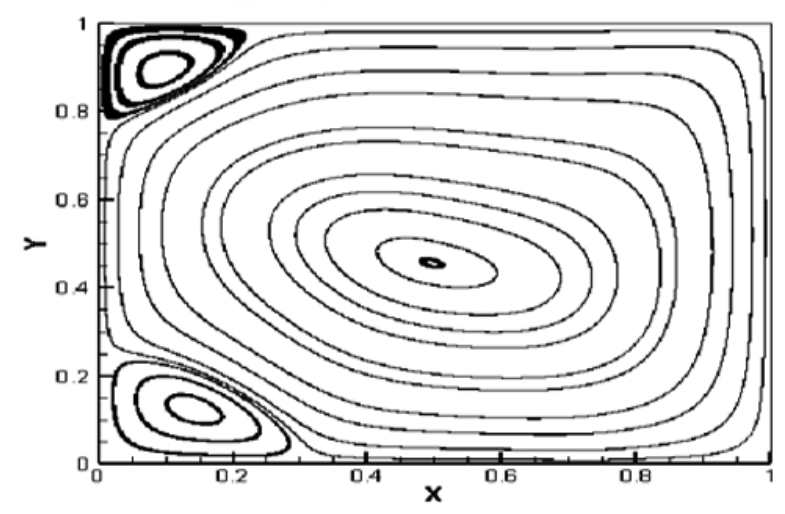

(c)

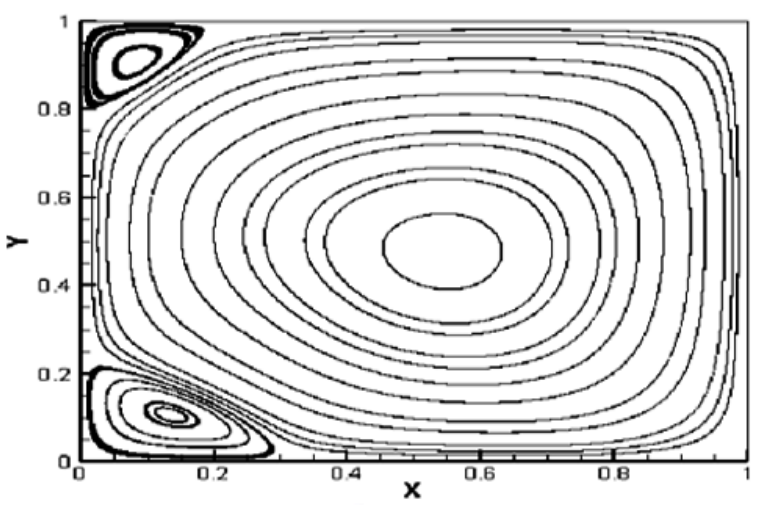

(b)

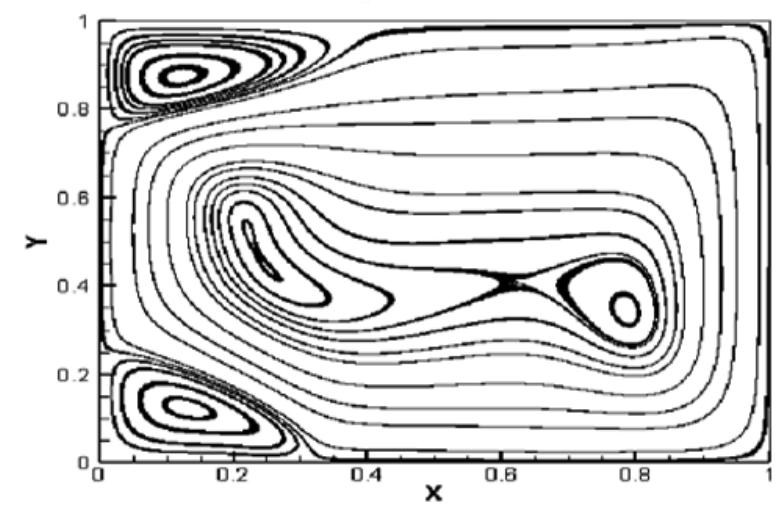

(d)

Fig. 5 Stream lines for different $\mathrm{RaN}$ for $\Phi=15$ percent volume fraction of $\mathrm{Al}_{2} \mathrm{O}_{3}$ nanoparticles. (a) $\mathrm{RaN}=10^{3}$, (b) $\mathrm{RaN}=10^{4}$, (c) $\mathrm{RaN}=10^{5}$, (d) $\mathrm{RaN}=10^{6}$

$\mathrm{RaN}=10^{3}$, maximum horizontal velocity component increased by $19 \%$ with $5 \%$ addition of nanoparticles and thereafter decreased by $5 \%, 25 \%$ and $36 \%$ than base fluid velocity with increase in nanoparticle volume fraction from $10 \%$ to $20 \%$. Similarly, at $\mathrm{RaN}=10^{4}$ as it can be seen in Fig. 7 (b), nanofluid maximum horizontal velocity component increased by $28 \%$ with $5 \%$ volume fraction and then starts to decrease by $26 \%, 21 \%$ and $15 \%$ with further addition of nanoparticles up to $20 \%$ due to increase in density and viscosity of nanofluids. It was also observed that nanofluid velocities were higher than base fluid velocities due to the effect of two heated sources on adjacent surfaces. From the Fig. 7 (c) at $\mathrm{RaN}=10^{5}$, the maximum horizontal velocity component of nanofluids was observed to be increased upon addition of volume fraction of nanoparticles from $5 \%$ to $20 \%$ than the base fluid by $22 \%, 34 \%, 43 \%$ and $50 \%$ due to increase in the buoyancy forces at high RaN. And also, from the Fig. 7 (d) at $\mathrm{RaN}=10^{6}$, maximum horizontal velocity component shows $20 \%$ increase in velocity for $5 \%$ addition and decreases by $14 \%$ and $12 \%$ for $10 \%$ and $15 \%$ addition of nanoparticles and again increases to $16 \%$ for $20 \%$ volume fraction of nanoparticles. From Fig. 7 (a) to (d) in can also be observed that variation of horizontal component of velocity, $U$, along the $Y$ direction changes form symmetry to asymmetry due to formation of multi circular vortices at high $\mathrm{RaN}$ in the central region of the cavity.

\subsection{Impact of volume fraction of nanoparticles on vertical component of velocity, $V$}

The influence of volumetric percentage addition of $\mathrm{Al}_{2} \mathrm{O}_{3}$ nanoparticles on vertical velocity components, $V$, along the mid horizontal line inside the square cavity at different $\mathrm{RaN}$ is shown in Fig. 8. As it is illustrated in Fig. 8 (a), at low $\mathrm{RaN}=10^{3}$, the maximum vertical velocity component increased by $21 \%$ with $5 \%$ addition of nanoparticles and thereafter decreased by $1 \%, 18 \%$ and $30 \%$ than base fluid velocity with further increase in volume fraction of nanoparticles from $10 \%$ to $20 \%$. Similarly at $\mathrm{RaN}=10^{4}$ as shown in Fig. 8 (b), nanofluid's maximum vertical velocity component increased by $31 \%$ with $5 \%$ volume fraction and then starts to decrease by $24 \%, 17 \%$ and 


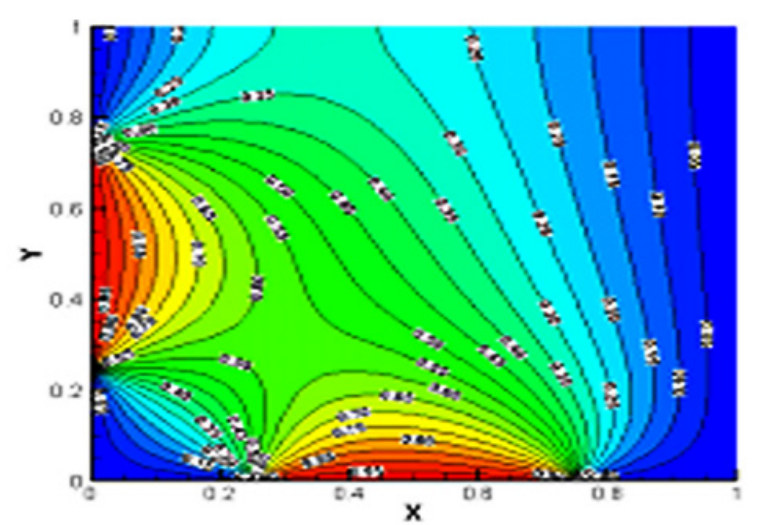

(a)

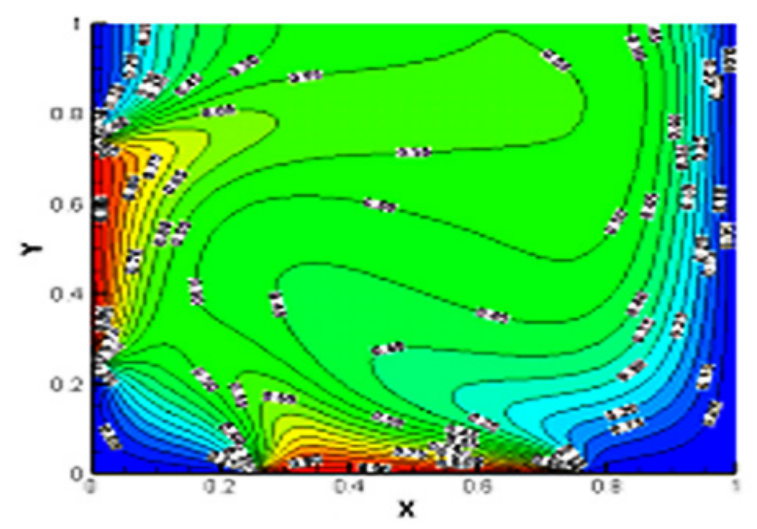

(c)

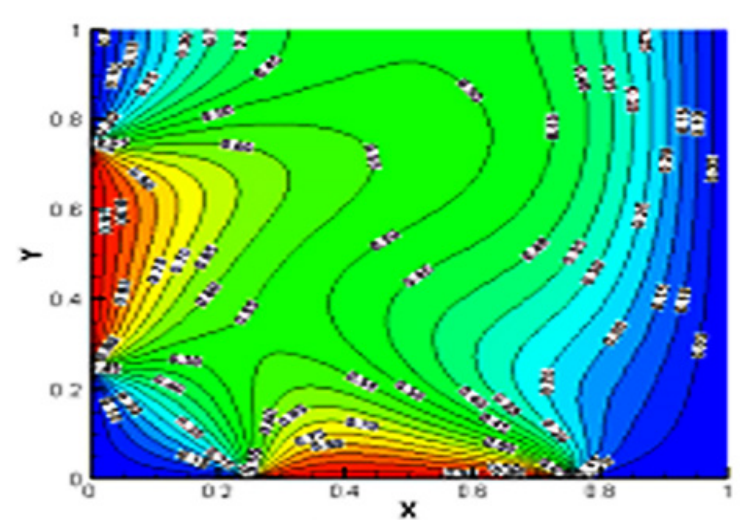

(b)

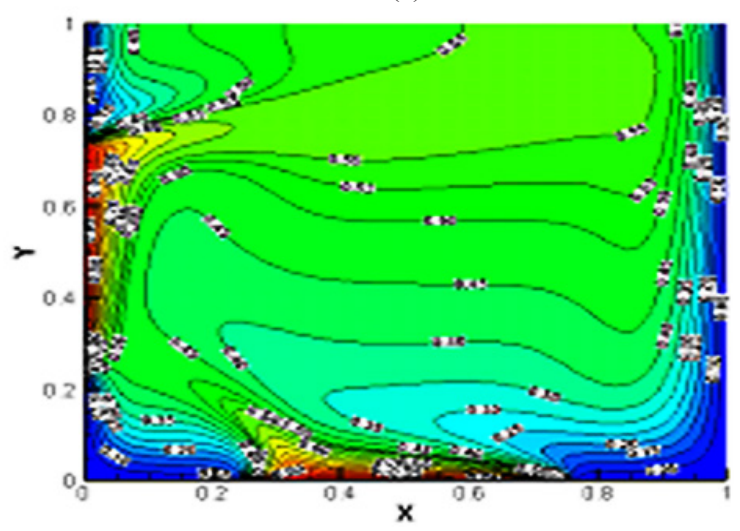

(d)

Fig. 6 Isothermallines for different $\operatorname{RaN}$ for $\Phi=15$ percent volume fraction of nanoparticles. (a) $\mathrm{RaN}=10^{3},(\mathrm{~b}) \mathrm{RaN}=10^{4}$, (c) $\mathrm{RaN}=10^{5}$, (d) $\mathrm{RaN}=10^{6}$

$9 \%$ on further addition of nanoparticles up to $20 \%$ due to increase in density and viscosity of nanofluids. It also observed that the nanofluid velocities were more than the base fluid velocities. From the Fig. 8 (c) at $\mathrm{RaN}=10^{5}$, it can be noticed that the maximum vertical velocity component of nanofluids was decreased upon addition of volume fraction of nanoparticles from $5 \%$ to $20 \%$ by $27 \%$, $24 \%$, $21 \%$ and $17 \%$ but it is more than the base fluid velocity. And also, from the Fig. 8 (d) at $\mathrm{RaN}=10^{6}$, vertical velocity became nearly zero in the central region of cavity and it was maximum near the vertical surfaces, whereas maximum vertical velocity component decreased on addition of nanoparticles from $5 \%$ to $20 \%$ by $23 \%, 22 \%, 21 \%$ and $18 \%$ respectively.

\subsection{Impact of volume fraction of nanoparticles and RaN on local Nusselt number}

The impact of volume fraction of nanoparticles and RaN on local Nusselt number along the bottom localized heated surfaces is illustrated in Fig. 9 (a) to (d). As it can be seen, with the increase in volume fraction of nanoparticles, the shape of local Nusselt number variation did not change but its magnitude increased with increase in RaN. For low RaN, the variation of local Nusselt number was symmetrical and for high RaN of $10^{5}$ and $10^{6}$ it exhibited asymmetry. Also, $\mathrm{Nu}_{\text {loc }}$ increased with percentage increase in volume fraction of nanoparticles and this was due to increase in thermal conductivity of nanofluid and also due to high convective heat transfer rates at high RaN of $10^{5}$ and $10^{6}$. Similarly Fig. 10 (a) to (d) shows the distribution of $\mathrm{Nu}_{\text {loc }}$ along the left surface for different RaN and volume fractions of nanoparticles. It also exhibits same variation for local Nusselt number but with lesser intensity which resulted in lower rate of heat transfer over the left surface compared with bottom heated surface.

4.6 Impact of volume fraction of $\mathrm{Al}_{2} \mathrm{O}_{3}$-nanoparticles in a base fluid and $\mathrm{RaN}$ on $\mathrm{Nu}_{\text {avg }}$ along the bottom surface Fig. 11 illustrates the variation in $\mathrm{Nu}_{\text {avg }}$ along the bottom surface of square cavity filled with $\mathrm{NF}$ of $\mathrm{Al}_{2} \mathrm{O}_{3}$ Water at different volume fractions of nanoparticles (from $5 \%$ to $20 \%$ ) for different $\mathrm{RaN}$ ranging from $10^{3}$ to $10^{6}$. 


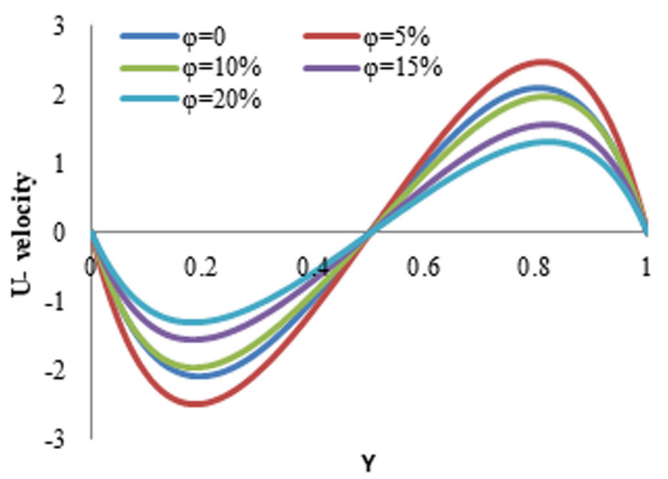

(a)

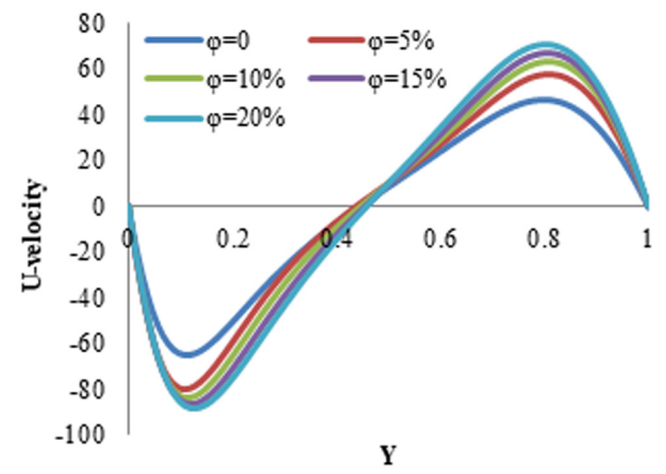

(c)

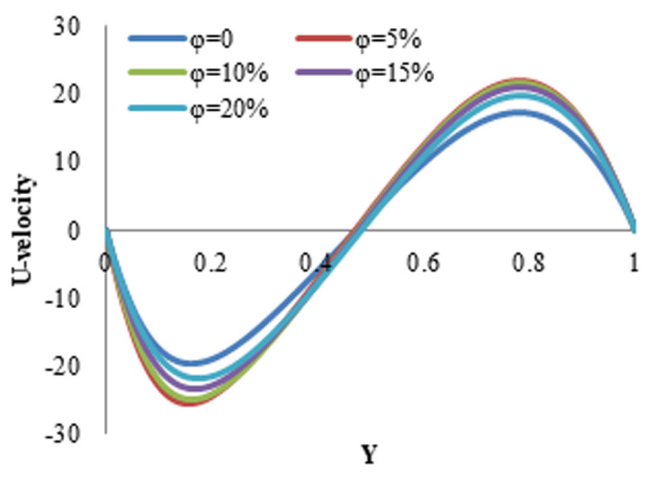

(b)

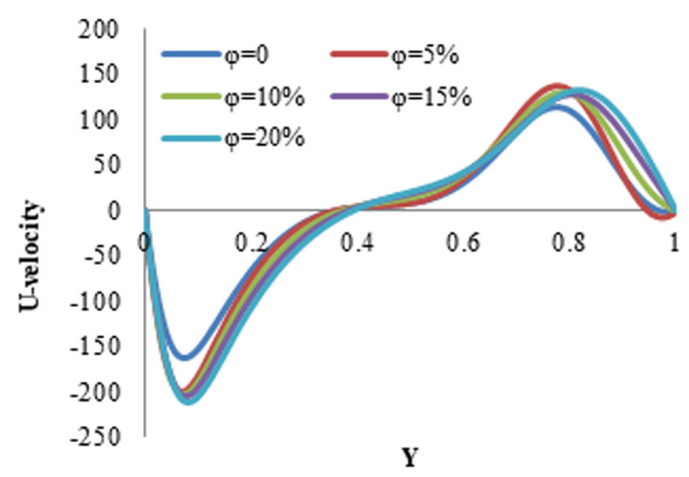

(d)

Fig. 7 Change of horizontal component of velocity, $U$, along the mid vertical line of square cavity by varying RaN from $10^{3}$ to $10^{6}$ for different volume percentage addition of $\mathrm{Al}_{2} \mathrm{O}_{3}$-nanoparticles. (a) $\mathrm{RaN}=10^{3}$, (b) $\mathrm{RaN}=10^{4}$, (c) $\mathrm{RaN}=10^{5}$, (d) $\mathrm{RaN}=10^{6}$

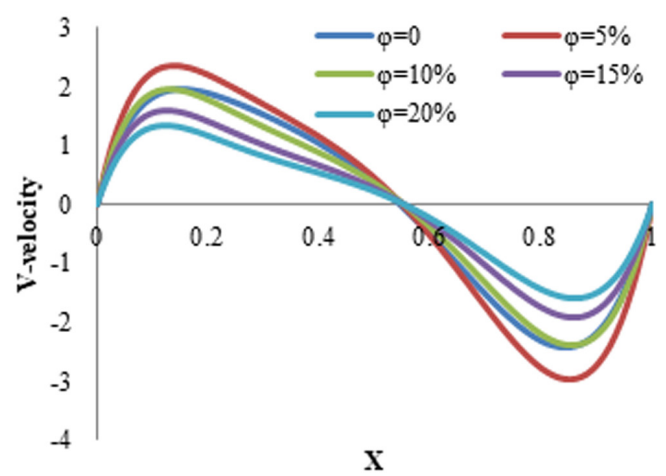

(a)

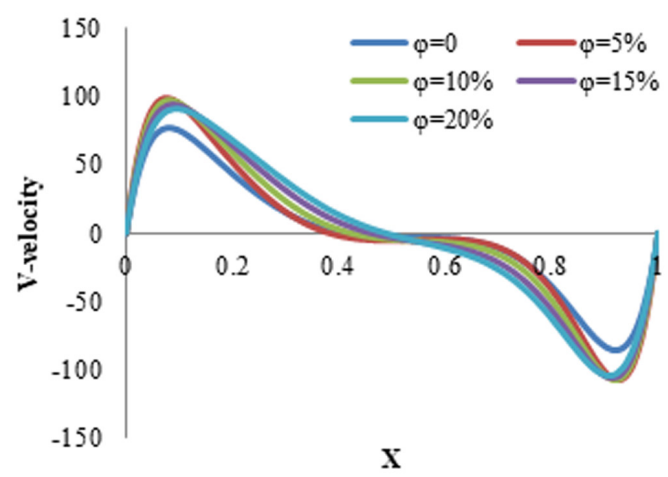

(c)

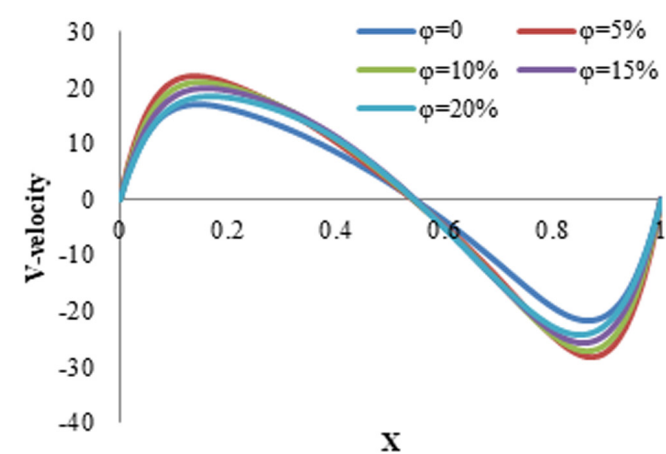

(b)

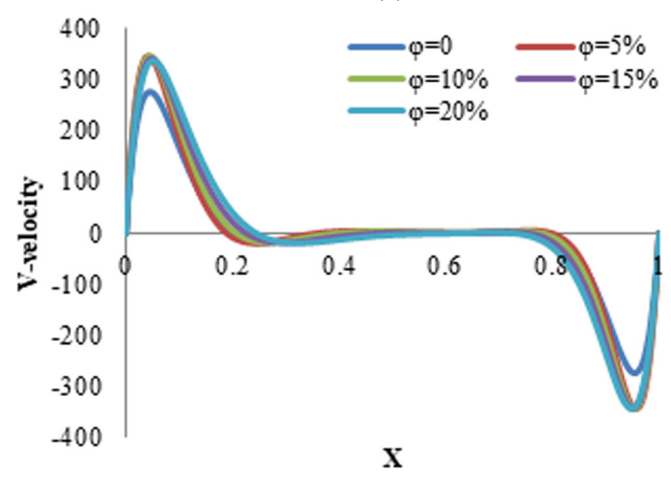

(d)

Fig. 8 Change of vertical component of velocity, $V$, along the mid vertical line of square cavity by varying RaN from $10^{3}$ to $10^{6}$ for different volume percentage addition of $\mathrm{Al}_{2} \mathrm{O}_{3}$-nanoparticles. (a) $\mathrm{RaN}=10^{3}$, (b) $\mathrm{RaN}=10^{4}$, (c) $\mathrm{RaN}=10^{5}$, (d) $\mathrm{RaN}=10^{6}$ 


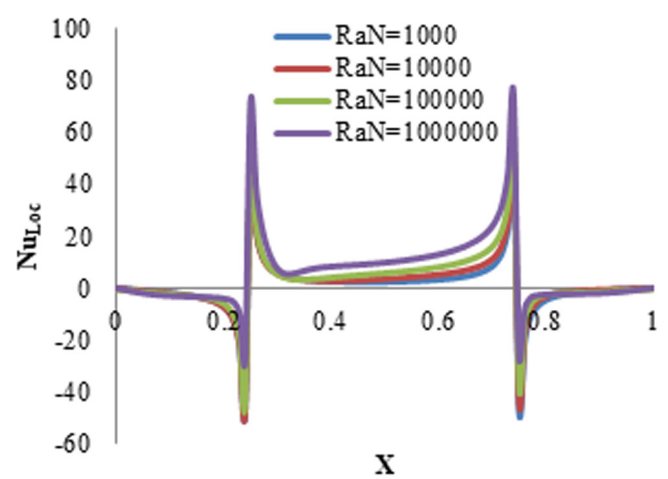

(a)

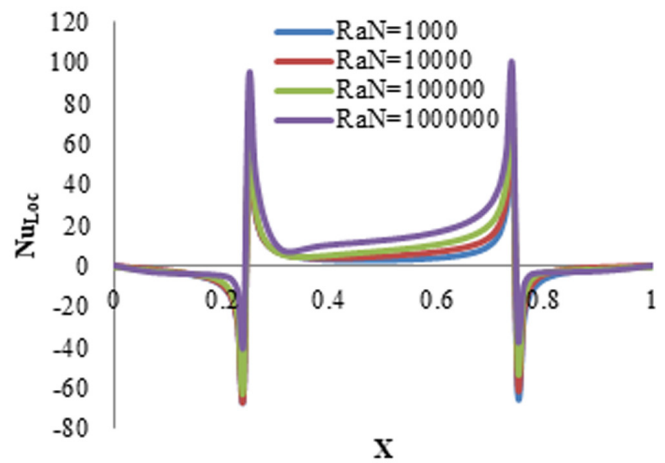

(c)

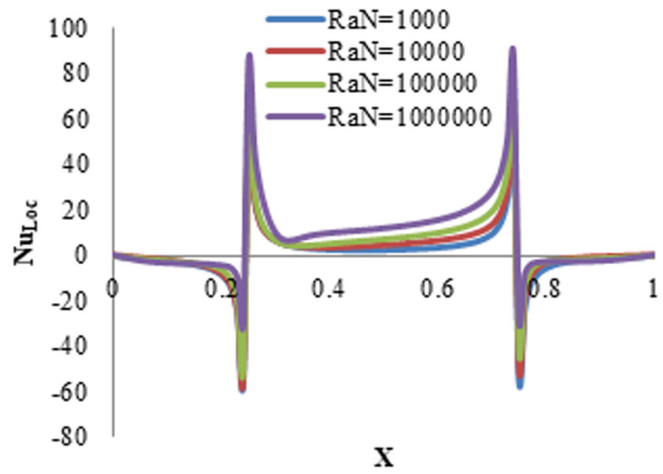

(b)

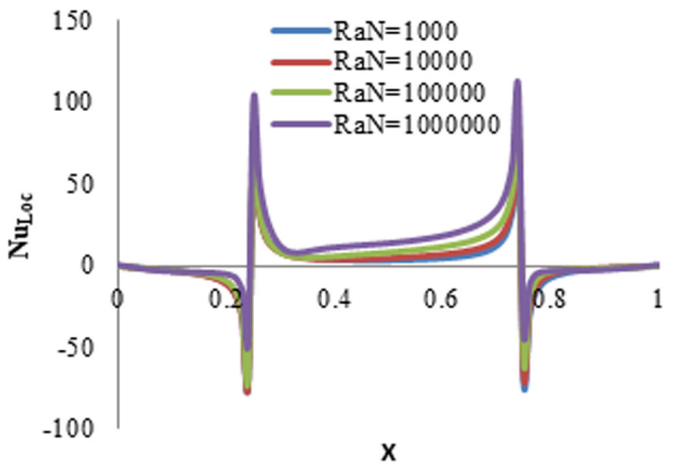

(d)

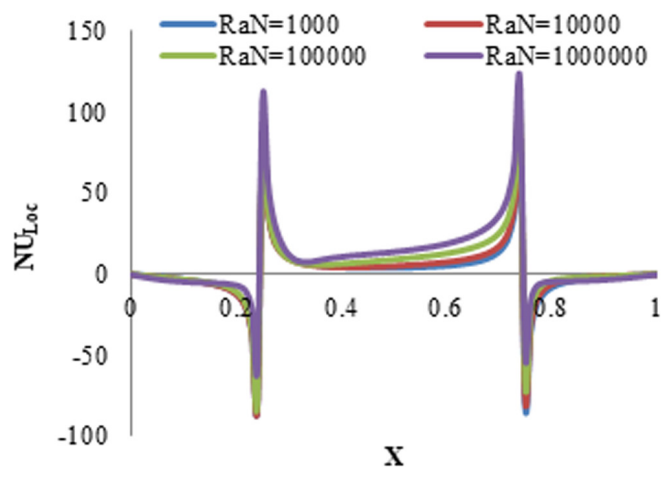

(e)

Fig. 9 Change of local Nusselt number along the bottom surface of square cavity for different volume fractions of $\mathrm{Al}_{2} \mathrm{O}_{3}$-nanoparticles and $\mathrm{RaN}$ (a) $\Phi=0 \%$, (b) $\Phi=5 \%$, (c) $\Phi=10 \%$, (d) $\Phi=15 \%$, (e) $\Phi=20 \%$

The addition of nanoparticles to the base fluid causes thermal conductivity of NF to be higher than base fluid and this will be proportional to the volume percentage addition of nanoparticles. For low $\operatorname{RaN}\left(\operatorname{RaN}=10^{3}\right.$ and $\left.10^{4}\right)$, there was a slight increase in $\mathrm{Nu}_{\text {avg }}$ due to the dominant effect of conduction mode of heat transfer over the convection at the bottom heated surface. The convective heat transfer was found to be dominant over the conduction heat transfer with the increase in $\mathrm{RaN}$ (from $\mathrm{RaN}=10^{5}$ and $10^{6}$ ) and volume fraction of nanoparticles in the base fluid. This is due to the increase in buoyancy forces inside the cavity which resulted in significant increase in heat transfer along the bottom surface.

\subsection{Impact of volume fraction of $\mathrm{Al}_{2} \mathrm{O}_{3}$-nanoparticles in} a base fluid and $\mathrm{RaN}$ on $\mathrm{Nu}_{\text {avg }}$ along the left surface

The variation of $\mathrm{Nu}_{\text {avg }}$ along the left heated surface is shown in Fig. 12. From $\mathrm{RaN}=1000$ to $10^{6}, \mathrm{Nu}_{\text {avg }}$ increases with increase in volume fraction of nanoparticles but with lesser intensity than on bottom heated surface. This is due to the fact that nanofluid gets heated as it first flows over the bottom heated surface and there exists less temperature difference as it comes in contact with left heated surface, which results in lower heat transfer rate. But at $\mathrm{RaN}=10^{6}, \mathrm{Nu}_{\text {avg }}$ again shows slight increasing trend due to the higher fluid velocities experienced by nanofluids as a result of increase in buoyancy forces. 


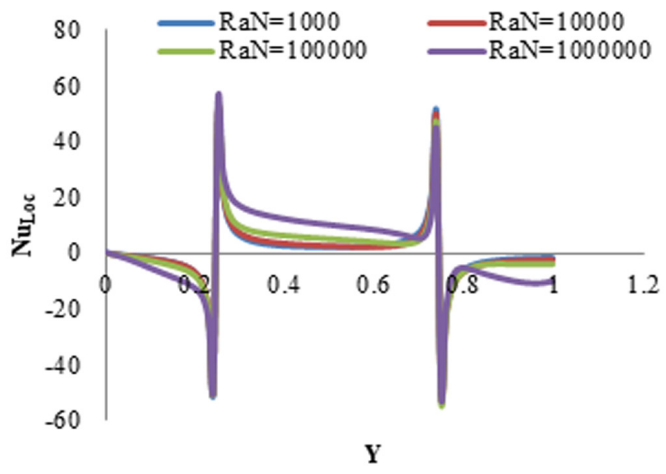

(a)

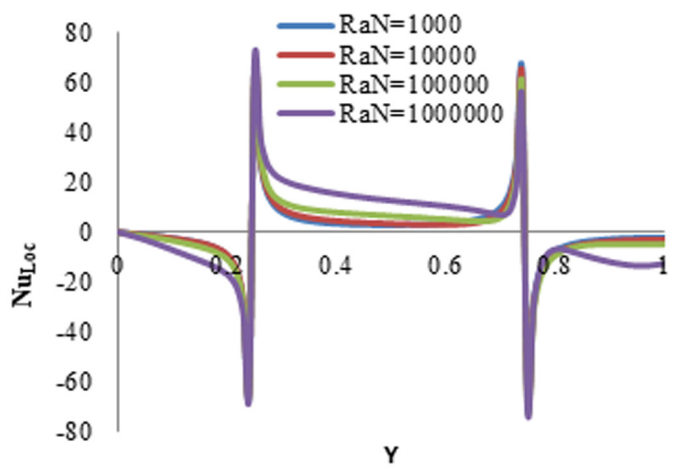

(c)

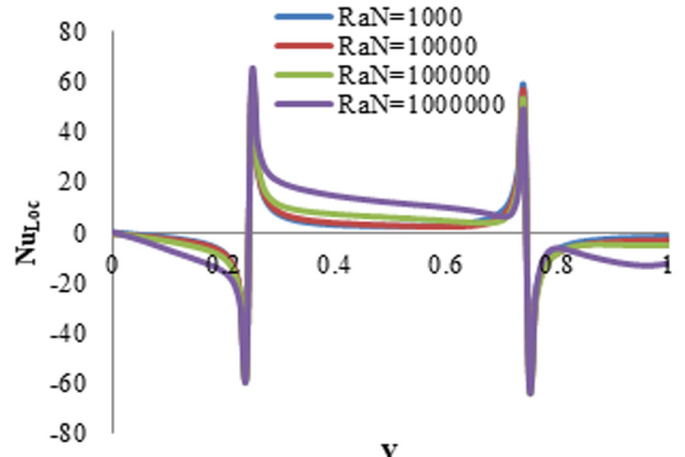

(b)

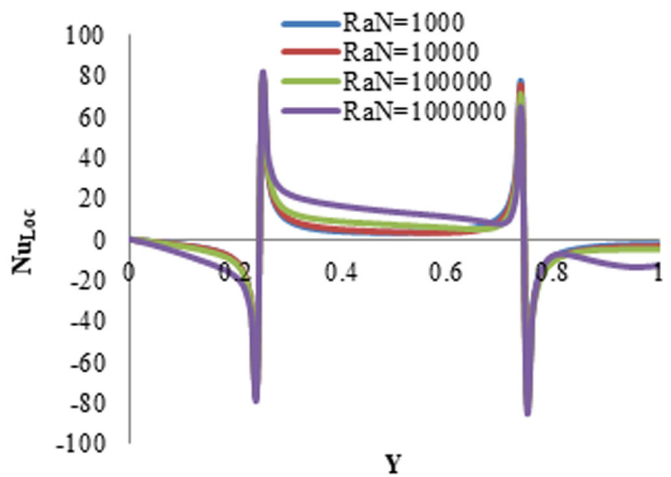

(d)

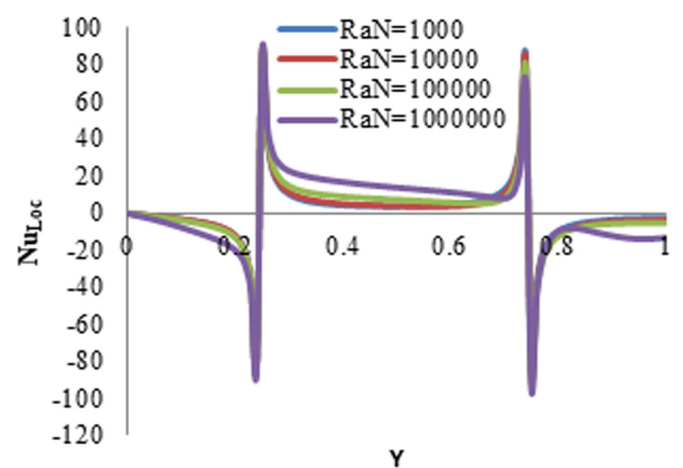

(e)

Fig. 10 Change of local Nusselt number along the left surface of square cavity for different volume fractions of $\mathrm{Al}_{2} \mathrm{O}_{3}$-nanoparticles and RaN.

(a) $\Phi=0 \%$, (b) $\Phi=5 \%$, (c) $\Phi=10 \%$, (d) $\Phi=15 \%$, (e) $\Phi=20 \%$

\section{Conclusion}

This paper investigates the impact of $\mathrm{RaN}$ and volume fractions of nanoparticles in a square cavity filled with $\mathrm{Al}_{2} \mathrm{O}_{3}$-Water NF on fluid flow and heat transfer characteristics using finite volume method and SIMPLE algorithm. The important conclusions from the study are presented below:

1. With the increase in $\mathrm{RaN}$ from $10^{3}$ to $10^{6}$, there was a significant change in fluid flow patterns and streamlines from single circulating vortices to multi circulating vortices in the center of square cavity.

2. At low RaN, poor heat transfer to nanofluid was observed due to conduction heat transfer near the heated surfaces and it was found to be dominating the convection.

3. There was a significant increase in both horizontal and vertical component of velocities in the mid lines of square cavity with increase in $\mathrm{RaN}$ and volume fraction of nanoparticles.

4. The increase in $\mathrm{Nu}_{\text {avg }}$ along the bottom surface was found to be higher than left heated surface with the increase in RaN and volume fraction of nanoparticles in the base fluid.

5. The distribution of $\mathrm{Nu}_{\text {loc }}$ variation over the heated surface was observed to be symmetrical for low $\operatorname{RaN}\left(10^{3}\right.$ and $\left.10^{4}\right)$ due to low fluid velocities and 


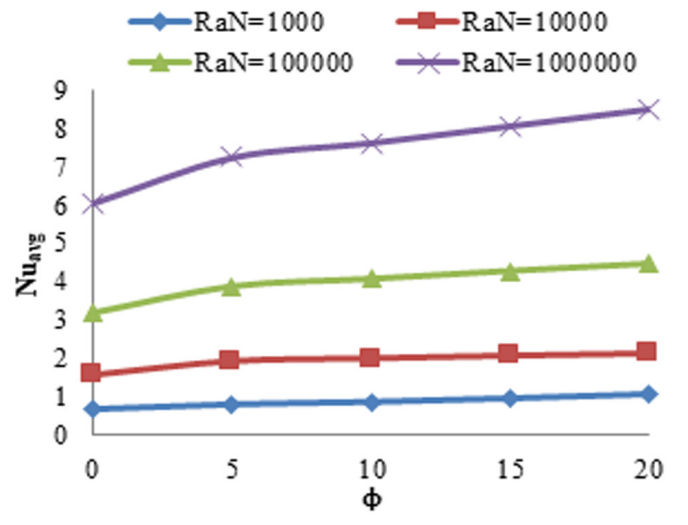

Fig. 11 Comparison of $\mathrm{Nu}_{\text {avg }}$ along the bottom surface with increase in volume fraction of $\mathrm{Al}_{2} \mathrm{O}_{3}$-particles for $\mathrm{RaN}$ varying from $10^{3}$ to $10^{6}$.

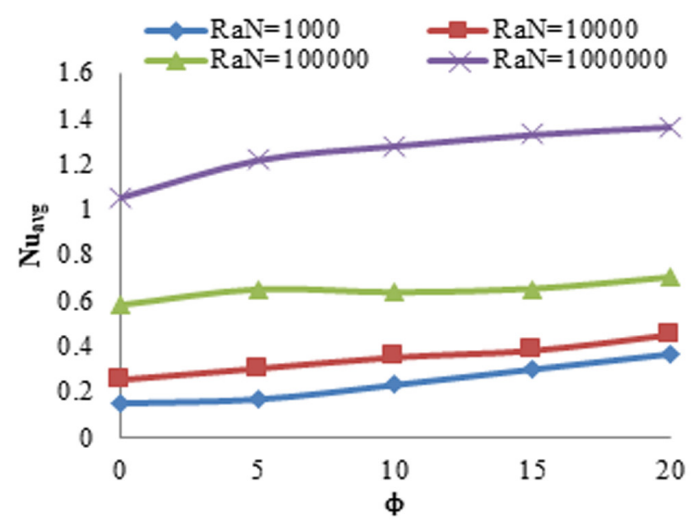

Fig. 12 Comparison of $\mathrm{Nu}_{\text {avg }}$ along the left surface with increase in volume fraction of $\mathrm{Al}_{2} \mathrm{O}_{3}$-particles for $\mathrm{RaN}$ varying from $10^{3}$ to $10^{6}$.

domination of heat conduction, and it was unsymmetrical for high $\operatorname{RaN}\left(10^{5}\right.$ and $\left.10^{6}\right)$ due to high fluid velocities and higher convective heat transfer rates.

\section{Acknowledgement}

The research is out of independent research of the authors and it is not funded by any organization. The authors declare that they have no conflict of interest.

\section{References}

[1] Choi, S. U. S., Eastman, J. A. "Enhancing thermal conductivity of fluids with nanoparticles", presented at 1995 International Mechanical Engineering Congress and Exhibition, San Francisco, CA, USA, Nov., 12-17, 1995.

[2] Keblinski, P., Phillpot, S. R., Choi, S. U. S., Eastman, J. A. "Mechanisms of heat flow in suspensions of nano-sized particles (nanofluids)", International Journal of Heat and Mass Transfer, 45(4), pp. 855-863, 2002.

https://doi.org/10.1016/S0017-9310(01)00175-2

[3] Xuan, Y., Li, Q. "Heat transfer enhancement of nanofluids", International Journal of Heat and Fluid Flow, 21(1), pp. 58-64, 2000.

https://doi.org/10.1016/S0142-727X(99)00067-3

\section{Nomenclature}

$C \quad$ specific heat, $(\mathrm{J} / \mathrm{Kg} \mathrm{K})$

$g$ acceleration due to gravity, $\left(\mathrm{m} / \mathrm{s}^{2}\right)$

$k$ thermal conductivity, (W/m K)

NFS nanofluids

$\mathrm{Nu}_{\text {loc }} \quad$ local Nusselt number

$\mathrm{Nu}_{\text {avg }}$ average Nusselt number

Pr Prandtl number

$p \quad$ fluid pressure, $(\mathrm{Pa})$

$P \quad$ non-dimensional pressure

RaN Rayleigh number

$T$ dimensional temperature, $(\mathrm{K})$

$u, v \quad$ dimensional $x$ and $y$ components of velocity, $\left(\mathrm{ms}^{-1}\right)$

$U, V \quad$ dimensionless velocities

$U_{\max }, \quad$ dimensionless maximum velocities

$V_{\max }$

$x, y \quad$ dimensional coordinates, $(\mathrm{m})$

$X, Y \quad$ dimensionless coordinates

$L \quad$ Length of the cavity, (m)

Greek symbols

$\alpha \quad$ thermal diffusivity, $\left(\mathrm{m}^{2} / \mathrm{s}\right)$

$\beta \quad$ thermal expansion coefficient, $(1 / \mathrm{K})$

$\Phi \quad$ nanoparticles volume fraction

$\theta \quad$ non dimensional temperature

$\mu \quad$ dynamic viscosity, $\left(\mathrm{N} \mathrm{s} / \mathrm{m}^{2}\right)$

$v \quad$ kinematic viscosity, $\left(\mathrm{m}^{2} / \mathrm{s}\right)$

$\rho \quad$ density, $\left(\mathrm{kg} / \mathrm{m}^{3}\right)$

Subscripts

$\begin{array}{ll}c & \text { cold surface } \\ f & \text { fluid } \\ h & \text { hot surface } \\ n f & \text { nanofluid } \\ p & \text { solid particles }\end{array}$

[4] Jang, S. P., Choi, S. U. S. "Free Convection in a Rectangular Cavity (Benard Convection) With Nanofluids", In: ASME International Mechanical Engineering Congress and Exposition, Anaheim, CA, USA, 2004, pp. 147-153 https://doi.org/10.1115/IMECE2004-61054

[5] Jang, S. P., Choi, S. U. S. "Role of Brownian motion in the enhanced thermal conductivity of nanofluids", Applied Physics Letters, 84(21), pp. 4316-4318, 2004. https://doi.org/10.1063/1.1756684

[6] Xie, H., Wang, J., Xi, T., Liu, Y., Ai, F., Wu, Q. "Thermal conductivity enhancement of suspensions containing nanosized alumina particles", Journal of Applied Physics, 91(7), pp. 4568-4572, 2002. https://doi.org/10.1063/1.1454184 
[7] Wang, G., Zhang, Y., Zhang, J., Ma, B. "Effects of Nanoparticles Shape and Concentration on Natural Convection of $\mathrm{Al}_{2} \mathrm{O}_{3}$ Water Nanofluid in a Cubic Enclosure", Chemical Engineering Transactions, 61, pp. 1171-1176, 2017.

https://doi.org/10.3303/CET1761193

[8] Khalili, E., Saboonchi, A., Saghafian, M. "Natural Convection of $\mathrm{Al}_{2} \mathrm{O}_{3}$ Nanofluid Between Two Horizontal Cylinders Inside a Circular Enclosure", Heat Transfer Engineering, 38(2), pp. 177-189, 2017. https://doi.org/10.1080/01457632.2016.1177382

[9] Bondarenko, D. S., Sheremet, M. A., Oztop H. F., Ali, M. E. "Natural convection of $\mathrm{Al}_{2} \mathrm{O}_{3} / \mathrm{H}_{2} \mathrm{O}$ nanofluid in a cavity with a heat-generating element. Heatline visualization", International Journal of Heat and Mass Transfer, 130, pp. 564-574, 2019. https://doi.org/10.1016/j.ijheatmasstransfer.2018.10.091

[10] Rashidi, I., Mahian, O., Lorenzini, G., Biserni, C., Wongwises, S. "Natural convection of $\mathrm{Al}_{2} \mathrm{O}_{3}$ /water nanofluid in a square cavity: Effects of heterogeneous heating", International Journal of Heat and Mass Transfer, 74, pp. 391-402, 2014.

https://doi.org/10.1016/j.ijheatmasstransfer.2014.03.030
[11] Maxwell, J. C. "A Treatise on Electricity and Magnetism", Cambridge University Press, Cambridge, UK, 2010. https://doi.org/10.1017/CBO9780511709340

[12] Brinkman, H. C. "The Viscosity of Concentrated Suspensions and Solutions", The Journal of Chemical Physics, 20(4), Article number: $571,1952$. https://doi.org/10.1063/1.1700493

[13] De Vahl Davis, G. "Natural convection of air in a square cavity: A bench mark numerical solution", International Journal for Numerical Methods in Fluids, 3(3), pp. 249-264, 1983. https://doi.org/10.1002/fld.1650030305

[14] Markatos, N. C., Pericleous, K. A. "Laminar and turbulent natural convection in an enclosed cavity", International Journal of Heat and Mass Transfer, 27(5), pp. 755-772, 1984. https://doi.org/10.1016/0017-9310(84)90145-5 